\title{
CLOSURES OF REGULAR LANGUAGES FOR PROFINITE TOPOLOGIES
}

\author{
J. ALMEIDA, J. C. COSTA, AND M. ZEITOUN \\ Dedicated to the memory of John M. Howie
}

\begin{abstract}
The Pin-Reutenauer algorithm gives a method, that can be viewed as a descriptive procedure, to compute the closure in the free group of a regular language with respect to the Hall topology.

A similar descriptive procedure is shown to hold for the pseudovariety $A$ of aperiodic semigroups, where the closure is taken in the free aperiodic $\omega$-semigroup. It is inherited by a subpseudovariety of a given pseudovariety if both of them enjoy the property of being full. The pseudovariety A, as well as some of its subpseudovarieties are shown to be full.

The interest in such descriptions stems from the fact that, for each of the main pseudovarieties $\mathrm{V}$ in our examples, the closures of two regular languages are disjoint if and only if the languages can be separated by a language whose syntactic semigroup lies in $\mathrm{V}$. In the cases of $\mathrm{A}$ and of the pseudovariety DA of semigroups in which all regular elements are idempotents, this is a new result.
\end{abstract}

\section{INTRODUCTION}

One of the motivations of this paper can be formulated as the following separation problem: for a fixed variety of regular languages [30], is it decidable whether two given regular languages can be separated by one from the chosen variety? A classical example of such a variety is that of star-free languages. We approach this question using algebraic and topological tools: Eilenberg's correspondence associates to any variety of regular languages a pseudovariety of semigroups. For instance, the pseudovariety corresponding to the variety of all star-free languages is that of aperiodic semigroups. In turn, to each pseudovariety $\mathrm{V}$, one can associate a topological semigroup $\bar{\Omega}_{X} \mathrm{~V}$. It is generated by the alphabet $X$, in the sense that every element of $\bar{\Omega}_{X} \mathrm{~V}$ is the limit of a sequence of words. Furthermore, there is a canonical mapping $X^{+} \rightarrow \bar{\Omega}_{X} \vee$, so that we can view any $L \subseteq X^{+}$as a subset of $\bar{\Omega}_{X} \mathrm{~V}$. The connection with the separation problem is the following: for a given

Date: January 2, 2014.

2010 Mathematics Subject Classification. Primary 20M07; Secondary 20M05, 20M35, $68 \mathrm{Q} 70$.

Key words and phrases. pseudovariety, profinite semigroup, profinite topology, pointlike set, regular language, aperiodic semigroup, topological closure. 
pseudovariety $\mathrm{V}$ of semigroups, two regular languages of $X^{+}$can be separated by a language recognized by $\mathrm{V}$ if and only if the intersection of their topological closures in $\bar{\Omega}_{X} \vee$ is empty [1, Corollary 3.6.5]. This perspective was first addressed in [3] with the aim of computing joins of pseudovarieties. A similar approach was also carried out with a broader scope of applications in $[35,36$.

The difficulty lies in computing inside $\bar{\Omega}_{X} \mathrm{~V}$, which may be an uncountable semigroup whose structure depends on $\mathrm{V}$. This motivates the definition of reducibility of a pseudovariety $\mathrm{V}$, introduced by Steinberg and the first author [12, that we use under a restricted form. Informally, it means that testing emptiness of such an intersection of closures in $\bar{\Omega}_{X} \mathrm{~V}$ can be done in a smaller and more manageable subsemigroup of $\bar{\Omega}_{X} \mathrm{~V}$, whose elements can be represented by finite terms over a suitable signature. Therefore, to answer the separation problem, it is relevant to compute closures of regular languages in this subsemigroup. The main contribution of this paper is an algorithm to compute a finite representation for such closures.

To compute the closure of a regular language in the free group, Pin and Reutenauer [32] proposed an iterative descriptive procedure: namely, they conjectured that the closure operator commutes with finite unions and products, while the closure of $L^{*}$, for some regular language $L$, is the subgroup of the free group generated by $L$. In view of classical results on rational subsets of the free group, this yields an algorithm for computing the closure of a regular language. The conjecture has been established by Ash [17, whose inevitability theorem entails the stronger conjecture of Pin and Reutenauer that any finite product of finitely generated subgroups of the free group is closed. In an equivalent form [10, 11], Ash's theorem was rediscovered by Herwig and Lascar [25]. The stronger conjecture, equivalent to the Rhodes type II conjecture [23, was also proved by Ribes and Zalesskii [34. An elementary and constructive proof was obtained recently by Auinger 18 (see also [19]). It is worth noting that Ash's inevitability theorem, as well as the Ribes and Zalesskil theorem also imply that two regular languages can be separated by a group language if and only if their closures in the free group are disjoint. Thus the Pin-Reutenauer procedure solves the separation problem with respect to the pseudovariety $\mathrm{G}$ of finite groups.

In this paper, we establish that the Pin-Reutenauer iterative descriptive procedure also holds for the pseudovarieties $\mathrm{A}$ of finite aperiodic semigroups, DA of finite semigroups in which all regular elements are idempotents, J of finite J-trivial semigroups, LSI of finite local semilattices, and $\mathrm{R}$ of finite $\mathcal{R}$-trivial semigroups. For each of these pseudovarieties $\mathrm{V}$, the semigroup in which the closures of regular languages are computed is the smallest subsemigroup of $\bar{\Omega}_{X} \vee$ containing $X$ and closed under $\omega$-power. Since $A$ is reducible in the restricted form mentioned above [22, 24], and J, R and LSI are reducible (even in a more general sense [4, 7, 21]), the results of this paper reduce the separation problem for each of these pseudovarieties to testing emptiness of intersections of closures of regular languages as described by 
the Pin-Reutenauer iterative descriptive procedure. Reducibility of DA will be established in a forthcoming paper [6].

The paper is organized as follows: we recall the definition and basic properties of profinite semigroups in Section 2. We formulate the main results in Section 3. The behavior of the closure operator with respect to the language operations of concatenation and Kleene plus, which are the key ingredients in the Pin-Reutenauer descriptive procedure, are analyzed in Section 4 for an arbitrary pseudovariety. The relationship of the concatenation case with factoriality properties is investigated in Section 5 . The necessary previous results on the pseudovariety $A$ are recalled in Section 6, leading to Proposition 6.5, which states that, for a regular language $L$, the closure of $L^{+}$is the subsemigroup closed under $\omega$-power generated by $L$. In Section 7, several pseudovarieties, including $A$, are shown to have a key property known as $\omega$-fullness, which relates the desired closure with the closure in the profinite semigroup $\bar{\Omega}_{X} \mathrm{~V}$.

\section{BACKGROUND AND NOTATION}

We refer the reader to [5] for an introduction to the theory of profinite semigroups, or to [1, 33] for more comprehensive treatments.

Recall that a semigroup pseudovariety is a class of finite semigroups closed under taking finite direct products, subsemigroups and quotients. We write $S$ for the pseudovariety of all finite semigroups and $A$ for that of all finite aperiodic (that is, group-free) semigroups. Throughout the paper, we fix a finite alphabet $X$. For a pseudovariety $\vee$, we denote by $\bar{\Omega}_{X} \vee$ the pro- $\vee$ semigroup freely generated by $X$. We shall freely use the fact that $\bar{\Omega}_{X} \vee$ is a metric space. Elements of $\bar{\Omega}_{X} \mathrm{~V}$ are called pseudowords over $\mathrm{V}$. Each $u \in \bar{\Omega}_{X} \mathrm{~V}$ can be naturally interpreted in a pro- $\mathrm{V}$-semigroup $T$, by the mapping $u_{T}: T^{X} \rightarrow T$ which associates to each function $\varphi: X \rightarrow T$ the element $\hat{\varphi}(u) \in T$, where $\hat{\varphi}$ is the unique extension of $\varphi$ to a continuous homomorphism from $\bar{\Omega}_{X} \vee$ to $T$. For instance, if $X=\{a, b\}$, the interpretation of $u=a b$ is the semigroup multiplication in $T$. For an element $u$ of a pro- $V$ semigroup, $u^{\omega}$ denotes the unique idempotent in the closed subsemigroup generated by $u$.

An implicit signature is a set $\sigma$ of pseudowords containing the semigroup multiplication. A pro- $\mathrm{V}$ semigroup $T$ has a natural structure of $\sigma$-semigroup, that is, a structure of $\sigma$-algebra in which each operation in $\sigma$ is naturally interpreted in $T$. Given an implicit signature $\sigma$, we denote by $\Omega_{X}^{\sigma} \mathrm{V}$ the $\mathrm{V}$-free $\sigma$-semigroup generated by $X$, whose elements are called $\sigma$-words (over $\mathrm{V}$ ). Each $\sigma$-word has a representation by a formal term over $X$ in the signature $\sigma$. These terms are called $\sigma$-terms. Sometimes, it will be convenient to use the following notation: given a $\sigma$-term $t$, denote by $[t]_{\vee}$ the $\sigma$-word over $\vee$, belonging to $\Omega_{X}^{\sigma} \mathrm{V}$, defined by $t$.

For example, for the canonical signature $\omega$ consisting of the multiplication and the $\omega$-power, $\omega$-terms are obtained from letters of $X$ using multiplication 
and $\omega$-power. Since our multiplication is associative, we identify terms that only differ by the order in which multiplications are to be carried out.

\section{The Pin-Reutenauer Procedure}

Given a pseudovariety $\mathrm{V}$, an implicit signature $\sigma$, and a subset $L$ of $\Omega_{X}^{\sigma} \mathrm{V}$, we denote

- by $\bar{L}$ the topological closure of $L$ in $\Omega_{X}^{\sigma} \mathrm{V}$, and

- by $\langle L\rangle$ the $\sigma$-subsemigroup of $\Omega_{X}^{\sigma} \mathrm{V}$ generated by $L$.

We say that the Pin-Reutenauer procedure holds for a pseudovariety $\mathrm{V}$ in the implicit signature $\sigma$ if, for all regular languages $K, L \subseteq X^{+}$, the following equations hold:

$$
\begin{aligned}
\overline{K L} & =\bar{K} \bar{L}, \\
\overline{L^{+}} & =\langle\bar{L}\rangle .
\end{aligned}
$$

This terminology is justified by the fact that the above formulas hold for the pseudovariety $\mathrm{G}$ of all finite groups, where the closures are computed in the free group [31, 32, 34].

The situation for groups turns out to be somewhat simpler, since the right side of formula (3.2) reduces to $\langle L\rangle$. To prove this, it suffices to observe that $L^{+}$is contained in $\langle L\rangle$, and that $\langle L\rangle$ is finitely generated by a theorem of Anissimow and Seifert [16] (see also [20, Thm. III.2.7]) and therefore it is closed by a theorem of M. Hall (see [32 for details). Since the AnissimowSeifert Theorem applies to an arbitrary group, a similar argument holds for every group pseudovariety $\mathrm{H}$ such that every finitely generated subgroup of the relatively free group over the pseudovariety $\mathrm{H}$ is closed.

We proceed with the statement of the main theorems, whose proofs are presented in the remainder of the paper. Our main contribution is to establish the Pin-Reutenauer procedure for certain pseudovarieties of finite aperiodic semigroups. The key step in this direction is to prove this property for the pseudovariety A, whose proof appears in Section 6, based on general results of Sections 4 and 5 .

Theorem 3.1. The Pin-Reutenauer procedure holds for the pseudovariety A with respect to the signature $\omega$.

We also prove that the Pin-Reutenauer procedure can be transferred from a pseudovariety to a subpseudovariety, provided both of them enjoy the property, introduced in 12, 13, of being $\sigma$-full. We shall show in Proposition 4.3 that a pseudovariety $\mathrm{V}$ is $\sigma$-full if and only if for every regular language $L \subseteq X^{+}$, the closure of $L$ in $\Omega_{X}^{\sigma} \mathrm{V}$ is the projection in $\Omega_{X}^{\sigma} \mathrm{V}$ of its closure in $\Omega_{X}^{\sigma} \mathrm{S}$.

Proposition 3.2. Let $\mathrm{V}$ and $\mathrm{W}$ be $\sigma$-full pseudovarieties such that $\mathrm{V} \subseteq \mathrm{W}$. If the Pin-Reutenauer procedure holds for $\mathrm{W}$ with respect to $\sigma$, then it also holds for V. 
An immediate consequence of Proposition 3.2 is that if the Pin-Reutenauer procedure holds for the pseudovariety $S$ of all finite semigroups with respect to the implicit signature $\sigma$ then it also holds for every $\sigma$-full pseudovariety. Indeed, $\mathbf{S}$ is trivially $\sigma$-full for every implicit signature $\sigma$.

Examples of application of Proposition 3.2 are derived from the following theorem, whose proof is the subject of Section 7 .

Theorem 3.3. The pseudovarieties A, DA, J, and $\mathrm{R}$ are $\omega$-full.

It has also been shown by Nogueira [28] that the $\omega$-fullness of the pseudovariety LSI follows immediately from the results in [21. Combining Theorem 3.1, Proposition 3.2 and Theorem 3.3 , we deduce the following result.

Corollary 3.4. The Pin-Reutenauer procedure holds for the pseudovarieties $\mathrm{DA}, \mathrm{J}, \mathrm{LSI}$ and $\mathrm{R}$ with respect to the signature $\omega$.

\section{BASIC PROPERTIES OF CLOSURES}

This section presents general results concerning closures of regular languages. It shows in particular that the Pin-Reutenauer procedure can be transferred from a pseudovariety to a subpseudovariety, provided both pseudovarieties are $\sigma$-full.

For a subset $L$ of a topological semigroup $T$, denote by $\mathrm{cl}_{T}(L)$ the closure of $L$ in $T$. We consider on $\Omega_{X}^{\sigma} \bigvee$ the induced topology as a subspace of $\bar{\Omega}_{X} \mathrm{~V}$. For convenience, we write $\mathrm{cl}(L)$ instead of $\mathrm{cl}_{\bar{\Omega}_{X} \mathrm{~S}}(L), \mathrm{cl}_{\sigma}(L)$ instead of $\operatorname{cl}_{\Omega_{X}^{\sigma} \mathrm{S}}(L)$, and $\mathrm{cl}_{\sigma, \mathrm{V}}(L)$ (or again $\bar{L}$ when $\sigma$ and $\mathrm{V}$ are understood) instead of $\operatorname{cl}_{\Omega_{X}^{\sigma} \mathrm{\vee}}(L)$. Note that, for every $L \subseteq \Omega_{X}^{\sigma} \mathrm{S}$,

$$
\operatorname{cl}_{\sigma}(L)=\operatorname{cl}(L) \cap \Omega_{X}^{\sigma} \mathrm{S} .
$$

Let $p_{\vee}: \bar{\Omega}_{X} \mathrm{~S} \rightarrow \bar{\Omega}_{X} \vee$ be the only continuous homomorphism sending each free generator to itself. Slightly abusing notation, for $L \subseteq X^{+}$, we write $\mathrm{cl}_{\sigma, \mathrm{V}}(L)$ to denote $\mathrm{cl}_{\sigma, \mathrm{\vee}}\left(p_{\mathrm{\vee}}(L)\right)$. This is harmless, since, for all pseudovarieties considered in this paper, the natural homomorphism from $X^{+}$into $\bar{\Omega}_{X} \bigvee$ is injective.

For a $\sigma$-semigroup $T$ and a subset $L$ of $T$, we denote by $\langle L\rangle_{\sigma}$ the $\sigma$ subsemigroup of $T$ generated by $L$. Finally, for $L \subseteq X^{+}$, we let $\langle L\rangle_{\sigma, \mathrm{V}}=$ $\left\langle p_{\vee}(L)\right\rangle_{\sigma}$ (or again $\langle L\rangle$ when $\sigma$ and $\mathrm{V}$ are understood), which is a subset of $\Omega_{X}^{\sigma} \mathrm{V}$.

Lemma 4.1. The following hold for a regular language $L$ of $X^{+}$:

(1) $\overline{L^{+}}$is a $\sigma$-subsemigroup of $\Omega_{X}^{\sigma} \mathrm{S}$;

(2) $\overline{L^{+}}=\overline{\langle L\rangle}$;

(3) $\langle\bar{L}\rangle \subseteq \overline{L^{+}}$.

Proof. Since $L$ is regular, so is $L^{+}$and by [1, Thm. 3.6.1], $\operatorname{cl}\left(L^{+}\right)$is a clopen subset of $\bar{\Omega}_{X} \mathrm{~S}$. Since the syntactic congruence of a clopen set is of finite index [29], there is a continuous homomorphism $\varphi: \bar{\Omega}_{X} \mathrm{~S} \rightarrow T$ onto a finite 
semigroup such that $\varphi^{-1} \varphi\left(\operatorname{cl}\left(L^{+}\right)\right)=\operatorname{cl}\left(L^{+}\right)$. Let $\psi$ be the restriction of $\varphi$ to $\Omega_{X}^{\sigma} \mathrm{S}$. Then,

$$
\begin{aligned}
\overline{L^{+}} & =\operatorname{cl}\left(L^{+}\right) \cap \Omega_{X}^{\sigma} \mathrm{S}=\varphi^{-1} \varphi\left(\operatorname{cl}\left(L^{+}\right)\right) \cap \Omega_{X}^{\sigma} \mathrm{S} \\
& =\psi^{-1} \varphi\left(\operatorname{cl}\left(L^{+}\right)\right)=\psi^{-1} \varphi\left(L^{+}\right)=\psi^{-1} \varphi(\langle L\rangle),
\end{aligned}
$$

where the next to last equality follows from the continuity of $\varphi$ and finiteness of $T$, and the last equality is a consequence of the fact that both $\varphi\left(L^{+}\right)$ and $\varphi(\langle L\rangle)$ coincide with the subsemigroup of $T$ generated by $\varphi(L)$, since $\varphi$ is a homomorphism of $\sigma$-semigroups. We deduce that $\operatorname{cl}_{\sigma}\left(L^{+}\right)$is a $\sigma$ subsemigroup of $\Omega_{X}^{\sigma} \mathrm{S}$, which proves (1).

For (2), note that the inclusion $L^{+} \subseteq\langle L\rangle$ implies $\overline{L^{+}} \subseteq \overline{\langle L\rangle}$. For the reverse inclusion, from $L \subseteq \overline{L^{+}}$, taking into account (1), we deduce that $\langle L\rangle \subseteq \overline{L^{+}}$, and then that $\overline{\langle L\rangle} \subseteq \overline{L^{+}}$since $\overline{L^{+}}$is closed.

Since $\bar{L} \subseteq \overline{L^{+}}$, the inclusion in (3) follows from (1).

Let $\mathrm{V}$ be a pseudovariety. Given a finite $X$-generated semigroup $T$ and an onto continuous homomorphism $\varphi: \bar{\Omega}_{X} \mathrm{~S} \rightarrow T$, we denote by $\mu_{\vee}$ the relational morphism $T \rightarrow \bar{\Omega}_{X} \vee$ given by $\mu_{\vee}=p_{\vee} \circ \varphi^{-1}$, by $\mu_{\vee}^{\sigma}$ the relational morphism $T \rightarrow \Omega_{X}^{\sigma} \vee$ given by $\mu_{\vee}^{\sigma}=p_{\vee} \circ\left(\left.\varphi\right|_{\Omega_{X}^{\sigma} \mathrm{S}}\right)^{-1}$, and by $\bar{\mu}_{\vee}^{\sigma}$ the relational morphism given by $\bar{\mu}_{\mathrm{V}}^{\sigma}=\mu_{\mathrm{V}} \cap\left(T \times \Omega_{X}^{\sigma} \mathrm{V}\right)$, which is therefore the topological closure of $\mu_{\mathrm{V}}^{\sigma}$ in $T \times \Omega_{X}^{\sigma} \mathrm{V}$. The following diagram summarizes the relationships between these homomorphisms and relational morphisms.

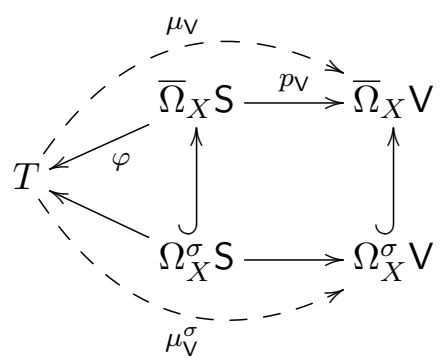

Following [12, 13], we say that $\mathrm{V}$ is $\sigma$-full if $\bar{\mu}_{\mathrm{V}}^{\sigma}=\mu_{\mathrm{V}}^{\sigma}$. Observe that, by definition, the pseudovariety $\mathrm{S}$ is trivially $\sigma$-full for every implicit signature $\sigma$. See [12, Table 2] and Section 7 for other examples of full pseudovarieties.

Lemma 4.2. Let $\mathrm{V}$ be a pseudovariety and $\sigma$ be an implicit signature. Then, for every language $L \subseteq X^{+}$, we have:

$$
\mathrm{cl}_{\sigma, \mathrm{\vee}}(L)=p_{\vee}(\operatorname{cl}(L)) \cap \Omega_{X}^{\sigma} \mathrm{\vee} .
$$

Proof. To prove the inclusion from left to right, we observe that $p_{\vee}(L)$ is contained in the right side of equality (4.2) because $L \subseteq \operatorname{cl}(L) \cap X^{+}$. Since $\operatorname{cl}(L)$ is a closed subset of the compact space $\bar{\Omega}_{X} \mathrm{~S}$ and $p_{\mathrm{V}}: \bar{\Omega}_{X} \mathrm{~S} \rightarrow \bar{\Omega}_{X} \mathrm{~V}$ is continuous, it follows that $p_{\mathrm{V}}(\operatorname{cl}(L)) \cap \Omega_{X}^{\sigma} \mathrm{V}$ is a closed subset of $\Omega_{X}^{\sigma} \mathrm{V}$. Since it contains $p_{\mathrm{V}}(L)$, it also contains its closure $\mathrm{cl}_{\sigma, \mathrm{V}}(L)$.

For the reverse inclusion, let $w \in \operatorname{cl}(L)$ be such that $p_{\mathrm{\vee}}(w) \in \Omega_{X}^{\sigma} \vee$. Since $w \in \operatorname{cl}(L)$, there exists a sequence $\left(w_{n}\right)_{n}$ in $L$ converging to $w$. Since $p_{\vee}$ is 
continuous, we know that $p_{\mathrm{\vee}}(w)=\lim _{n} p_{\mathrm{\vee}}\left(w_{n}\right)$ and so $p_{\mathrm{\vee}}(w) \in \operatorname{cl}\left(p_{\mathrm{\vee}}(L)\right) \cap$ $\Omega_{X}^{\sigma} \mathrm{V}=\mathrm{cl}_{\sigma, \mathrm{V}}(L)$ by (4.1), which proves the statement.

We can now give several alternative characterizations of $\sigma$-fullness.

Proposition 4.3. The following are equivalent for a pseudovariety $\mathrm{V}$ and an implicit signature $\sigma$ :

(a) $\mathrm{V}$ is $\sigma$-full;

(b) for every regular language $L \subseteq X^{+}, p_{\vee}\left(\operatorname{cl}_{\sigma}(L)\right)=p_{\vee}(\operatorname{cl}(L)) \cap \Omega_{X}^{\sigma} \bigvee$;

(c) for every regular language $L \subseteq X^{+}, p_{\vee}\left(\operatorname{cl}_{\sigma}(L)\right)=\mathrm{cl}_{\sigma, \mathrm{\vee}}(L)$;

(d) for every regular language $L \subseteq X^{+}, p_{\mathrm{\vee}}\left(\mathrm{cl}_{\sigma}(L)\right)$ is closed in $\Omega_{X}^{\sigma} \mathrm{V}$.

Proof. The equivalence $(b) \Leftrightarrow(c)$ follows immediately from Lemma 4.2 .

Next, we note that the inclusion $p_{\mathrm{V}}\left(\mathrm{cl}_{\sigma}(L)\right) \subseteq \mathrm{cl}_{\sigma, \mathrm{V}}(L)$ is always true since $p_{\vee}\left(\operatorname{cl}_{\sigma}(L)\right)$ is certainly contained in the intersection $p_{\vee}(\operatorname{cl}(L)) \cap \Omega_{X}^{\sigma} \vee$, which in turn coincides with $\mathrm{cl}_{\sigma, \mathrm{V}}(L)$ by Lemma 4.2 . This yields $(c) \Leftrightarrow[(d)$,

It remains to prove $(c) \Leftrightarrow(a)$. Recall that $(a)$ means that, for every $X$ generated finite semigroup $T$, the two associated relational morphisms into $\Omega_{X}^{\sigma} \bigvee$ coincide: $\bar{\mu}_{\mathrm{V}}^{\sigma}=\mu_{\mathrm{V}}^{\sigma}$. Let $\varphi: X^{+} \rightarrow T$ be the homomorphism determined by the choice of generators and let $\hat{\varphi}: \bar{\Omega}_{X} \mathrm{~S} \rightarrow T$ be its unique continuous extension. Consider an arbitrary subset $P$ of $T$, and let $L=\varphi^{-1}(P)$. Note that $\hat{\varphi}^{-1}(P)=\operatorname{cl}(L)$ where the left to right inclusion is justified as follows: given $w \in \hat{\varphi}^{-1}(P)$, and a sequence $\left(w_{n}\right)_{n}$ in $X^{+}$converging to $w$, by continuity of $\hat{\varphi}$ and the fact that $T$ is discrete, we deduce that all but finitely many of the $w_{n}$ 's are in $\varphi^{-1}(P)$. Therefore, by definition of $\mu_{\mathrm{V}}^{\sigma}$ and Lemma 4.2 , we have:

$$
\mu_{\mathrm{V}}^{\sigma}(P)=p_{\mathrm{\vee}}\left(\operatorname{cl}(L) \cap \Omega_{X}^{\sigma} \mathrm{S}\right)=p_{\mathrm{\vee}}\left(\operatorname{cl}_{\sigma}(L)\right),
$$

and, by definition of $\bar{\mu}_{\mathrm{V}}^{\sigma}$ and Lemma 4.2 .

$$
\bar{\mu}_{\mathrm{V}}^{\sigma}(P)=p_{\vee}(\operatorname{cl}(L)) \cap \Omega_{X}^{\sigma} \vee=\operatorname{cl}_{\sigma, \mathrm{\vee}}(L)
$$

This yields the equivalence between $(c)$ and $(a)$.

We show that the condition that $L$ is regular of items $(b)+(d)$ of Proposition 4.3 cannot be dropped. Suppose that $a \in X$. Let $C=\left\{a^{2^{\alpha}}: \alpha \in\right.$ $\hat{\mathbb{N}}\} \subseteq \bar{\Omega}_{X} \mathrm{~S}$, where $\hat{\mathbb{N}}$ denotes the profinite completion of $\mathbb{N}$. We claim that $C$ is closed. Indeed, the equality $\hat{\mathbb{N}} \backslash\left\{2^{\alpha}: \alpha \in \hat{\mathbb{N}}\right\}=\bigcup_{p} p \hat{\mathbb{N}}$ holds, where the union runs over all odd primes; moreover, $p \hat{\mathbb{N}}$ is the preimage of 0 under the unique continuous homomorphism $\hat{\mathbb{N}} \rightarrow \mathbb{Z} / p \mathbb{Z}$ that sends 1 to 1 and, therefore $p \hat{\mathbb{N}}$ is open, whence $C$ is closed. On the other hand, we have $C \cap \Omega_{X}^{\omega} \mathrm{S}=C \cap X^{+}=\left\{a^{2^{n}} \mid n \in \mathbb{N}\right\}$ and $p_{\mathrm{A}}(C) \cap \Omega_{X}^{\omega} \mathrm{A}=p_{\mathrm{A}}\left(C \cap \Omega_{X}^{\omega} \mathrm{S}\right) \cup\left\{a^{\omega}\right\}$. Since $A$ is $\omega$-full, as shown later, the regularity assumption of items $(b)-(d)$ of Proposition 4.3 cannot therefore be dropped. This also shows that the mapping $p_{\mathrm{A}}: \Omega_{X}^{\omega} \mathrm{S} \rightarrow \Omega_{X}^{\omega} \mathrm{A}$ is not closed.

The following reformulation of Proposition 3.2 allows us to transfer properties (3.1) and (3.2) to subpseudovarieties, assuming fullness. 
Proposition 4.4. Let $\mathrm{V}, \mathrm{W}$ be two $\sigma$-full pseudovarieties such that $\mathrm{V} \subseteq \mathrm{W}$. Let $K, L \subseteq X^{+}$be regular languages.

(i) If $\mathrm{cl}_{\sigma, \mathrm{W}}(K L)=\mathrm{cl}_{\sigma, \mathrm{W}}(K) \mathrm{cl}_{\sigma, \mathrm{W}}(L)$ then $\mathrm{cl}_{\sigma, \mathrm{V}}(K L)=\mathrm{cl}_{\sigma, \mathrm{V}}(K) \mathrm{cl}_{\sigma, \mathrm{V}}(L)$.

(ii) If $\mathrm{cl}_{\sigma, \mathrm{W}}\left(L^{+}\right)=\left\langle\mathrm{cl}_{\sigma, \mathrm{W}}(L)\right\rangle_{\sigma}$ then $\mathrm{cl}_{\sigma, \mathrm{V}}\left(L^{+}\right)=\left\langle\mathrm{cl}_{\sigma, \mathrm{V}}(L)\right\rangle_{\sigma}$.

Proof. Denote by $p$ the canonical projection from $\bar{\Omega}_{X} \mathrm{~W}$ to $\bar{\Omega}_{X} \mathrm{~V}$. In the following calculations, we use freely the equivalence between properties $(c)$ and $(a)$ of Proposition 4.3 . Statement $(i)$ is obtained as follows:

$$
\begin{array}{rlrl}
\operatorname{cl}_{\sigma, \mathrm{V}}(K L) & =p \vee\left(\operatorname{cl}_{\sigma}(K L)\right) & & \text { since } \mathrm{V} \text { is } \sigma \text {-full } \\
& =p\left[p_{\mathrm{W}}\left(\mathrm{cl}_{\sigma}(K L)\right)\right] & & \text { since } \mathrm{W} \text { is } \sigma \text {-full } \\
& =p\left[\mathrm{cl}_{\sigma, \mathrm{W}}(K L)\right] & & \text { by hypothesis } \\
& =p\left[\operatorname{cl}_{\sigma, \mathrm{W}}(K) \mathrm{cl}_{\sigma, \mathrm{W}}(L)\right] & & \\
& =p\left[\mathrm{cl}_{\sigma, \mathrm{W}}(K)\right] p\left[\mathrm{cl}_{\sigma, \mathrm{W}}(L)\right] & & \\
& =p\left[p_{\mathrm{W}}\left(\mathrm{cl}_{\sigma}(K)\right)\right] p\left[p_{\mathrm{W}}\left(\mathrm{cl}_{\sigma}(L)\right)\right] & & \text { since } \mathrm{W} \text { is } \sigma \text {-full } \\
& =p \vee\left[\operatorname{cl}_{\sigma}(K)\right] p \mathrm{\vee}\left[\operatorname{cl}_{\sigma}(L)\right] & & \text { since } \mathrm{V} \text { is } \sigma \text {-full } \\
& =\mathrm{cl}_{\sigma, \mathrm{V}}(K) \mathrm{cl}_{\sigma, \mathrm{V}}(L) &
\end{array}
$$

Statement $(i i)$ is obtained similarly, taking additionally into account that $p_{\vee}$ is a homomorphism of $\sigma$-semigroups.

We conclude this section by observing that the topological closure on the right side of 3.2 cannot in general be dropped. Note that, since $\Omega_{X}^{\sigma} \mathrm{V}$ is a Hausdorff space, every finite subset of $\Omega_{X}^{\sigma} \mathrm{V}$ is certainly closed. Hence, if formula (3.2) holds, then every finitely generated $\sigma$-subsemigroup of $\Omega_{X}^{\sigma} \bigvee$ is closed.

Consider the regular language $L=a^{+} b^{+}$and its closure $\bar{L}$ in $\Omega_{X}^{\omega} \mathrm{S}$. We claim that $\langle L\rangle$ is not closed. Indeed, since $L^{+} \subseteq\langle L\rangle$, if $\langle L\rangle$ were closed then we would have $\overline{L^{+}} \subseteq\langle L\rangle$. Since $\langle L\rangle \subseteq\langle\bar{L}\rangle \subseteq \overline{L^{+}}$by Lemma 4.1, it would follow that $\langle L\rangle=\langle\bar{L}\rangle \supseteq \bar{L}$. Now, clearly $a^{\omega} b \in \bar{L}$, while for every element of $\langle L\rangle$ there is a bound on the exponents of its factors of the form $a^{n}$. Hence $\langle L\rangle$ is not closed in $\Omega_{X}^{\omega} \mathrm{S}$.

\section{Closure vs. Concatenation}

Proposition 3.2 motivates the problem of determining for which implicit signatures the Pin-Reutenauer procedure holds for S. In view of the above and later results in this paper, it would be particularly interesting to consider this problem for the signature $\omega$. In this section, we present a simple relationship between the behavior of the $\sigma$-closure operator with respect to the concatenation product of languages and an algebraic property of $\sigma$-words.

We say that a pseudovariety $\mathrm{V}$ is $\sigma$-factorial for an implicit signature $\sigma$ if, for every $(u, v) \in \Omega_{X}^{\sigma} \bigvee \times \bar{\Omega}_{X} \mathrm{~V}$, if $v$ is a factor of $u$, then $v \in \Omega_{X}^{\sigma} \mathrm{V}$. We next show that, for $\sigma$-factorial pseudovarieties, the topological closure and 
product commute for arbitrary languages (and not only for regular ones as is assumed for the Pin-Reutenauer procedure).

Proposition 5.1. Let $\sigma$ be an implicit signature, and let $\mathrm{V}$ be a $\sigma$-factorial pseudovariety. Then the following property holds:

$$
\text { for arbitrary } K, L \subseteq X^{+} \text {, we have } \overline{K L}=\bar{K} \bar{L} \text {. }
$$

Proof. Note that, for subsets $K, L \subseteq \Omega_{X}^{\sigma} \mathrm{V}$, the inclusion $\bar{K} \bar{L} \subseteq \overline{K L}$ is always true: it follows directly from the continuity of the multiplication. For the reverse inclusion, let $u \in \overline{K L}$ and consider a sequence $\left(u_{n}\right)_{n}$ from $K L$ converging to $u$. Let $u_{n}=v_{n} w_{n}$ with $v_{n} \in K$ and $w_{n} \in L$. Since $\bar{\Omega}_{X} \bigvee$ is compact, we may as well assume that the sequences $\left(v_{n}\right)_{n}$ and $\left(w_{n}\right)_{n}$ converge respectively to $v$ and $w$, whence $u=\lim u_{n}=v w$. By the assumption that $\mathrm{V}$ is $\sigma$-factorial, it follows that $v, w \in \Omega_{X}^{\sigma} \mathrm{V}$, so $v \in \bar{K}$ and $w \in \bar{L}$. Hence $u \in \bar{K} \bar{L}$.

The pseudovariety $\mathrm{V}$ is said to have infinite period if it satisfies no pseudoidentity of the form $x^{\omega+r}=x^{\omega}$ with $r$ a positive integer.

The following is a partial converse to Proposition 5.1.

Proposition 5.2. Let $\mathrm{V}$ be a pseudovariety satisfying the following conditions:

(a) property (5.1) holds;

(b) if $\mathrm{V}$ satisfies the pseudoidentity $x=y$ where $x$ is a word, then the pseudoidentity is trivial;

(c) $\vee$ has infinite period.

Then $\mathrm{V}$ is $\sigma$-factorial.

Proof. Let $u \in \Omega_{X}^{\sigma} \vee$ and $v, w \in \bar{\Omega}_{X} \bigvee$ be such that $u=v w$. We need to show that $v, w \in \Omega_{X}^{\sigma} \vee$. By $(b)$, if $u$ is a word from $X^{+}$, then so are $v$ and $w$. We therefore assume that $u \notin X^{+}$.

Let $\left(v_{n}\right)_{n}$ and $\left(w_{n}\right)_{n}$ be sequences of words converging in $\bar{\Omega}_{X} \mathrm{~V}$ to $v$ and $w$, respectively. In case $v$ (respectively $w$ ) does not belong to $X^{+}$, we may choose the corresponding sequence so that it consists of words of strictly increasing length. For $n \geq 0$, we set $K_{n}=\left\{v_{\ell}: \ell \geq n\right\}$ and $L_{n}=\left\{w_{\ell}: \ell \geq\right.$ $n\}$. Observe that $\overline{K_{n}} \subseteq K_{n} \cup\{v\}$ and $\overline{L_{n}} \subseteq L_{n} \cup\{w\}$. Since $u=v w \in \overline{K_{n} L_{n}}$, by condition (a) we deduce that $u \in \overline{K_{n}} \overline{L_{n}}$. Hence, there is a factorization $u=v_{n}^{\prime} w_{n}^{\prime}$ in $\sigma$-words with $v_{n}^{\prime} \in \overline{K_{n}} \subseteq K_{n} \cup\{v\}$ and $w_{n}^{\prime} \in \overline{L_{n}} \subseteq L_{n} \cup\{w\}$. So, if $v_{n}^{\prime}=v$ and $w_{n}^{\prime}=w$ for some $n$, then we are done. Otherwise, by symmetry and since $u \notin X^{+}$, we may assume that $v_{n}^{\prime} \in K_{n} \backslash\{v\}$ and $w_{n}^{\prime}=w$ for infinitely many values of $n$. We may further assume that $v \notin X^{+}$ for, otherwise, nothing remains to be proved. In particular, there are indices $m$ and $n$ such that $m<n, v_{m}^{\prime}, v_{n}^{\prime} \in X^{+}$, and $u=v_{m}^{\prime} w=v_{n}^{\prime} w$. Since the lengths of the words $v_{m}^{\prime}$ and $v_{n}^{\prime}$ are not equal, there is some letter $a \in X$ which occurs a different number of times in them. Substituting $a^{\omega}$ for every other letter in $v_{m}^{\prime} w=v_{n}^{\prime} w$, we obtain an equality of the form $a^{\alpha+k}=a^{\alpha+\ell}$ 
for some $\alpha \in \hat{\mathbb{N}}$ and some distinct positive integers $k$ and $\ell$. This is in contradiction with condition $(c)$. Hence, both $v$ and $w$ belong to $\Omega_{X}^{\sigma} \mathrm{V}$.

Note that $\mathrm{S}$ cannot be $\sigma$-factorial if $\sigma$ is countable and contains some pseudoword which is not a word. Indeed, the infinite powers of a letter in $\bar{\Omega}_{X} \mathrm{~S}$ form an uncountable group, while $\Omega_{X}^{\sigma} \mathrm{S}$ is countable. Since properties $(b)$ and (c) of Proposition 5.2 hold for $\mathrm{S}$, it follows that (5.1) fails for $\mathrm{V}=\mathrm{S}$ and such a signature $\sigma$. This is the case in particular for $\sigma=\omega$.

\section{THE APERIODIC CASE}

The aim of this section is to introduce the required tools and results on $\omega$-words over A for the proof of Theorem 3.1.

The rank of an $\omega$-term is the maximum number of nested applications of the $\omega$-power. By the rank of an $\omega$-word $w$ we mean the smallest rank of $\omega$-term $u$ representing $w$.

If the $\omega$-term $u$ is a word, define $E_{n}(u)=\{u\}$. For $u=v_{0} u_{1}^{\omega} v_{1} \cdots u_{r}^{\omega} v_{r}$, where all the $u_{j}$ have the same rank $i$ and all the $v_{j}$ have rank at most $i$, we let $E_{n}(u)=\left\{v_{0} u_{1}^{n_{1}} v_{1} \cdots u_{r}^{n_{r}} v_{r}: n_{1}, \ldots, n_{r} \geq n\right\}$. Then, for an $\omega$-term $u$, we let $L_{n}(u)=\left(E_{n}\right)^{\operatorname{rank}(u)}(u)$, that is, we iterate $\operatorname{rank}(u)$ times the operator $E_{n}$. For instance, for $u=\left(a^{\omega} b\right)^{\omega}$, of rank 2 , we have $L_{n}(u)=\left(a^{n} a^{*} b\right)^{n}\left(a^{n} a^{*} b\right)^{*}$.

McCammond [26] has shown that the word problem is effectively solvable in $\Omega_{X}^{\omega} \mathrm{A}$ by finding a rewriting system reducing any $\omega$-term to a normal form and showing that distinct $\omega$-terms in normal form cannot represent the same element of $\Omega_{X}^{\omega} \mathrm{A}$. An alternative proof of uniqueness of the normal form representative was obtained by the authors [8]. The main ingredient is the property stated in the following theorem. This property also plays a key role in the proof of the results of this section. The exact definition of the normal form is not relevant for our present purposes and so we refer the interested reader to [26].

Theorem 6.1 ([8, Theorem 5.1]). Let $u$ be an $\omega$-term in normal form. Then, for $n$ large enough, the language $L_{n}(u)$ is star-free.

The following two corollaries are simple consequences of Theorem 6.1.

Corollary 6.2 ([8, Corollary 7.2]). If $u$ is an arbitrary $\omega$-term, then

$$
p_{\mathrm{A}}\left(\bigcap_{n} \operatorname{cl}\left(L_{n}(u)\right)\right)=\left\{[u]_{\mathrm{A}}\right\}=\bigcap_{n} p_{\mathrm{A}}\left(\operatorname{cl}\left(L_{n}(u)\right)\right) .
$$

Corollary 6.3 ([8, Theorem 7.3]). Let $u$ be an w-term in normal form. Then $p_{\mathrm{A}}^{-1}\left([u]_{\mathrm{A}}\right)=\bigcap_{n} \operatorname{cl}\left(L_{n}(u)\right)$.

Another key property of the pseudovariety $A$ is given by the following theorem.

Theorem 6.4 ([8, Theorem 7.4]). The pseudovariety A is $\omega$-factorial. 
In view of Proposition 5.1, it follows from Theorem 6.4 that the operator $\mathrm{cl}_{\omega, \mathrm{A}}(-)$ behaves well with respect to concatenation of arbitrary languages.

To complete the proof of Theorem 3.1, it remains to treat the case of the closure of regular languages of the form $L^{+}$, as done in Proposition 6.5 below. The rest of the section is devoted to the proof of this result.

Proposition 6.5. If $L \subseteq X^{+}$is a regular language then $\operatorname{cl}_{\omega, \mathrm{A}}\left(L^{+}\right) \subseteq$ $\left\langle\mathrm{cl}_{\omega, \mathrm{A}}(L)\right\rangle_{\omega}$.

Proof. Let $w$ be an $\omega$-term in normal form such that $[w]_{\mathrm{A}} \in \mathrm{cl}_{\omega, \mathrm{A}}\left(L^{+}\right)$. By Theorem 6.1. for $n$ large enough, the set $p_{\mathrm{A}}\left(\operatorname{cl}\left(L_{n}(w)\right)\right)$ is clopen in $\bar{\Omega}_{X} \mathrm{~A}$. Since it contains $[w]_{\mathrm{A}}$ by Corollary 6.3 , the hypothesis that $[w]_{\mathrm{A}} \in \operatorname{cl}_{\omega, \mathrm{A}}\left(L^{+}\right)$ implies that there exists $w_{n} \in L^{+} \cap L_{n}(w)$. Note that $\left(w_{n}\right)_{n}$ converges to $[w]_{\mathrm{A}}$ in $\bar{\Omega}_{X} \mathrm{~A}$ by Corollary 6.2 . We write

$$
w_{n}=w_{n, 1} \cdots w_{n, r_{n}}
$$

where each $w_{n, i}$ is a word in $L$.

Let $\alpha_{0} \beta_{1}^{\omega} \alpha_{1} \cdots \beta_{k}^{\omega} \alpha_{k}$ be the normal form factorization of $w$, in which the factors $\beta_{i}^{\omega} \alpha_{i} \beta_{i+1}^{\omega}$ are crucial portions and the factors $\alpha_{0} \beta_{1}^{\omega}$ and $\beta_{k}^{\omega} \alpha_{k}$ are respectively initial and final portions in the sense of [26]. Thus, we have $\operatorname{rank}\left(\alpha_{i}\right) \leq \operatorname{rank}\left(\beta_{j}\right)=\operatorname{rank}(w)-1$. The proof proceeds by induction on the pair of parameters $(\operatorname{rank}(w), k)$, ordered lexicographically, where we postpone for now the treatment of the case $k=1$. Note that the case where $\operatorname{rank}(w)=0$ is trivial, since then $w \in L^{+} \subseteq\left\langle\operatorname{cl}_{\omega, \mathrm{A}}(L)\right\rangle_{\omega}$.

The condition $w_{n} \in L_{n}(w)$ means that there is a factorization

$$
w_{n}=\alpha_{0, n} \beta_{1, n} \alpha_{1, n} \cdots \beta_{k, n} \alpha_{k, n}
$$

where each $\alpha_{i, n} \in L_{n}\left(\alpha_{i}\right)$ and each $\beta_{i, n} \in L_{n}\left(\beta_{i}^{\omega}\right)$. In particular, each $\beta_{i, n}=\beta_{i, n, 1} \cdots \beta_{i, n, s_{i, n}}$ is a product of $s_{i, n} \geq n$ factors $\beta_{i, n, \ell}$ from $L_{n}\left(\beta_{i}\right)$. Note that $\lim \alpha_{i, n}=\left[\alpha_{i}\right]_{\mathrm{A}}$ and $\lim \beta_{i, n, j}=\left[\beta_{i}\right]_{\mathrm{A}}$ by Corollary 6.2 .

Comparing the two factorizations (6.1) and (6.2) of $w_{n}$, each index $i$ with $1 \leq i \leq r_{n}-1$ determines a factorization

$$
w_{n}=w_{n, 1} \cdots w_{n, i} \cdot w_{n, i+1} \cdots w_{n, r_{n}}
$$

and thus also a cut in the product on the right side of 6.2 into two factors. Such a cut takes place either within one of the factors $\beta_{j, n}$ or outside all of them. Moreover, a cut which falls in a factor $\beta_{j, n}$ will in turn determine a cut of some factor $\beta_{j, n, \ell}$.

Consider first the case where there is an infinite sequence $n_{1}<n_{2}<\cdots$ for which at least one of the indices $i_{p} \in\left\{1, \ldots, r_{n_{p}}-1\right\}$ determines a cut of the form (6.3), with $n=n_{p}$ and $i=i_{p}$, leaving intact a positive number of factors of the form $\beta_{j, n_{p}}$ on both sides of the cut. By the pigeonhole principle, we may assume that the cut always falls in the factors $\alpha_{j, n_{p}}$ for a fixed index $j \in\{1, \ldots, k-1\}$, or in some $\beta_{j, n_{p}, \ell_{p}}$, also with a fixed index $j \in\{2, \ldots, k-1\}$, but where $\ell_{p}$ may depend on $p$. In the first case, we have 
the equalities

$$
\begin{aligned}
w_{n_{p}, 1} \cdots w_{n_{p}, i_{p}} & =\alpha_{0, n_{p}} \beta_{1, n_{p}} \alpha_{1, n_{p}} \cdots \beta_{j, n_{p}} x_{p} \\
w_{n_{p}, i_{p}+1} \cdots w_{n_{p}, r_{n_{p}}} & =y_{p} \beta_{j+1, n_{p}} \alpha_{j+1, n_{p}} \cdots \beta_{k, n_{p}} \alpha_{k, n_{p}} \\
x_{p} y_{p} & =\alpha_{j, n_{p}} .
\end{aligned}
$$

By compactness, we may assume that the sequences $\left(x_{p}\right)_{p}$ and $\left(y_{p}\right)_{p}$ converge, respectively, to $x$ and $y$. By Theorem 6.4 the pseudowords $x$ and $y$ satisfy $x=[\bar{x}]_{\mathrm{A}}$ and $y=[\bar{y}]_{\mathrm{A}}$ for some $\omega$-terms $\bar{x}$ and $\bar{y}$. We thus obtain the following factorization:

$$
[w]_{\mathrm{A}}=\left[\alpha_{0} \beta_{1} \alpha_{1} \cdots \beta_{j} \bar{x}\right]_{\mathrm{A}} \cdot\left[\bar{y} \beta_{j+1} \alpha_{j+1} \cdots \beta_{k} \alpha_{k}\right]_{\mathrm{A}} .
$$

From [9, Lemma 4.1], we deduce that $\operatorname{rank}(x)$ and $\operatorname{rank}(y)$ are at most rank $\left(\alpha_{j}\right)$, which in turn is less than rank $(w)$. By McCammond's normal form algorithm, it follows that the normal forms of the two factors on the right side of (6.6) have the same rank as $w$ and fewer $\omega$-factors of maximum rank, and so the induction hypothesis and equations (6.4) and (6.5) imply that those two factors belong to $\left\langle\mathrm{cl}_{\omega, \mathrm{A}}(L)\right\rangle_{\omega}$, whence so does $[w]_{\mathrm{A}}$.

The case where, for all $p$, all the cuts for some $i_{p}$ fall in some $\beta_{j, n_{p}, \ell_{p}}$ can be handled similarly and we leave the details to the reader. We may therefore consider only the case where, for each sufficiently large $n$, there is an index $i_{n} \in\left\{1, \ldots, r_{n}-1\right\}$ such that the factorizations (6.3), with $i=i_{n}$ and $i=i_{n}+1$ determine cuts respectively in $\alpha_{0, n} \beta_{1, n}$ and $\beta_{k, n} \alpha_{k, n}$ while the factor between those two cuts belongs to $\operatorname{cl}_{\omega, \mathrm{A}}(L)$. This achieves the announced reduction to the case where the parameter $k$ is 1 .

Hence, it suffices to consider the case where $w$ is of the form $w=\alpha \beta^{\omega} \gamma$ with $\max \{\operatorname{rank}(\alpha), \operatorname{rank}(\gamma)\} \leq \operatorname{rank}(\beta)=\operatorname{rank}(w)-1$. We then have two factorizations

$$
w_{n}=w_{n, 1} \cdots w_{n, r_{n}}=\alpha_{n} \beta_{n, 1} \cdots \beta_{n, m_{n}} \gamma_{n}
$$

of $w_{n} \in L_{n}(w)$, with the $w_{n, i} \in L, \alpha_{n} \in L_{n}(\alpha), \beta_{n, i} \in L_{n}(\beta), \gamma_{n} \in L_{n}(\gamma)$, $m_{n} \geq n$, and $\left(r_{n}\right)_{n}$ unbounded. By the previous induction scheme, we may further assume that $\alpha_{n}$ is a prefix of $w_{n, 1}$ and $\gamma_{n}$ a suffix of $w_{n, r_{n}}$.

Since $L$ is a regular language, there is a homomorphism $\varphi: X^{*} \rightarrow M$ onto a finite monoid $M$ such that $\varphi^{-1}(1)=\{1\}$ and $\varphi^{-1} \varphi(L)=L$. We construct for each $n$ a finite directed graph $\Gamma_{n}$. The set of vertices is

$$
V_{n}=\left\{(s, t) \in M \times M: L_{n}(\beta) \cap \varphi^{-1}(s) L^{*} \varphi^{-1}(t) \neq \emptyset\right\} \cup\left\{{ }^{\wedge}, \$\right\}
$$

where the two symbols ${ }^{\wedge}$ and $\$$ do not belong to $M$ and there is

- an edge $\left(s_{1}, t_{1}\right) \rightarrow\left(s_{2}, t_{2}\right)$ if

$$
L \cap \varphi^{-1}\left(t_{1}\right) L_{n}(\beta)^{*} \varphi^{-1}\left(s_{2}\right) \neq \emptyset,
$$

- an edge ${ }^{\wedge} \rightarrow(s, t)$ if

$$
L \cap L_{n}(\alpha) L_{n}(\beta)^{*} \varphi^{-1}(s) \neq \emptyset,
$$


- an edge $(s, t) \rightarrow \$$ if

$$
L \cap \varphi^{-1}(t) L_{n}(\beta)^{*} L_{n}(\gamma) \neq \emptyset .
$$

Note that, for each $n$, the factorizations (6.7) determine a path $\pi_{n}$ from vertex ${ }^{\wedge}$ to vertex $\$$. Figure 1 shows how these factorizations can overlap, where the factors mapped to values $s_{1}, t_{1}, s_{2}, t_{2}, \ldots$ in $M$ have been emphasized.

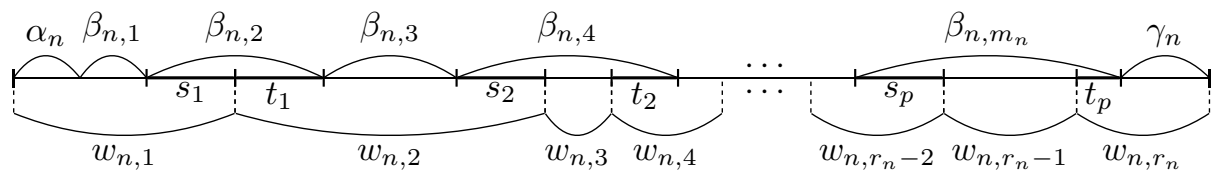

Figure 1. Overlapping factorizations and implied path

$$
\rightarrow\left(s_{1}, t_{1}\right) \rightarrow\left(s_{2}, t_{2}\right) \rightarrow \cdots \rightarrow\left(s_{p}, t_{p}\right) \rightarrow \$ \text { in } \Gamma_{n} \text { from 6.7) }
$$

Since $V_{n}$ is contained in the finite set $(M \times M) \cup\left\{{ }^{\wedge}, \$\right\}$, there is only a finite number of possibilities for the set $c\left(\pi_{n}\right)$ of the edges used in the path $\pi_{n}$. Hence there is a subsequence of $\left(w_{n}\right)_{n}$ for which the corresponding $c\left(\pi_{n}\right)$ is constant. By replacing each term in the sequence which does not appear in the subsequence by the first term in the subsequence that comes after it, we may as well assume that $c\left(\pi_{n}\right)$ is constant. We let $\Gamma$ be the subgraph of (all) $\Gamma_{n}$ whose vertices and edges are those that appear in $c\left(\pi_{n}\right)$.

We color each edge $\left(s_{1}, t_{1}\right) \rightarrow\left(s_{2}, t_{2}\right)$ in the graph $\Gamma$ with one of two colors:

- the edge is green whenever there is an unbounded sequence $\left(e_{n}\right)_{n}$ such that $\varphi^{-1}\left(t_{1}\right)\left(L_{n}(\beta)\right)^{e_{n}} \varphi^{-1}\left(s_{2}\right) \cap L \neq \emptyset$;

- otherwise, the edge is red.

To conclude the proof, consider two cases: (a) there is some green edge or (b) all edges are red.

In Case (a), we choose a path from the vertex ^ to the vertex $\$$ which includes some green edge, say the vertical edge in the following diagram (the cases where the green edge is the first or the last of the path are analogous):

$$
\begin{aligned}
\rightarrow\left(s_{1}, s_{2}\right) \rightarrow \cdots \rightarrow & \left(s_{2 k-1}, s_{2 k}\right) \\
\downarrow & \left(s_{2 k+1}, s_{2 k+2}\right) \rightarrow \cdots \rightarrow\left(s_{2 \ell-1}, s_{2 \ell}\right) \rightarrow \$ .
\end{aligned}
$$

By definition of the vertices of $\Gamma$, for each $n$ and each $i \in\{1, \ldots, \ell\}$, there exists $y_{n, i} \in L^{*}$ such that

$$
L_{n}(\beta) \cap \varphi^{-1}\left(s_{2 i-1}\right) y_{n, i} \varphi^{-1}\left(s_{2 i}\right) \neq \emptyset .
$$

Let $u_{n, 1}, \ldots, u_{n, 2 \ell}$ be words such that

$$
\left\{\begin{array}{l}
\varphi\left(u_{n, i}\right)=s_{i}, \\
u_{n, 2 i-1} y_{n, i} u_{n, 2 i} \in L_{n}(\beta) .
\end{array}\right.
$$


By definition of the edges of $\Gamma$, there exist integers $e_{n, i}$ such that all sets

$$
L \cap \varphi^{-1}\left(s_{2 i}\right)\left(L_{n}(\beta)\right)^{e_{n, i}} \varphi^{-1}\left(s_{2 i+1}\right)
$$

are nonempty for $i=1, \ldots, \ell-1$ and every $n$. Note that $\left(e_{n, k}\right)_{n}$ can be chosen unbounded, since the edge $\left(s_{2 k-1}, s_{2 k}\right) \rightarrow\left(s_{2 k+1}, s_{2 k+2}\right)$ is green. As $\varphi$ recognizes the language $L$ and as $u_{n, i} \in \varphi^{-1}\left(s_{i}\right)$, one can choose words

$$
x_{n, i} \in L \cap u_{n, 2 i}\left(L_{n}(\beta)\right)^{e_{n, i}} u_{n, 2 i+1} \text {, for } i \in\{1, \ldots, \ell-1\} .
$$

Similarly, there exist integers $e_{n, 0}$ and $e_{n, k}$ and words $x_{n, 0}$ and $x_{n, \ell}$ such that

$$
\begin{aligned}
& x_{n, 0} \in L \cap L_{n}(\alpha) L_{n}(\beta)^{e_{n, 0}} u_{n, 1} \\
& x_{n, \ell} \in L \cap u_{n, 2 \ell} L_{n}(\beta)^{e_{n, \ell}} L_{n}(\gamma) .
\end{aligned}
$$

Consider the word

$$
z_{n}=x_{n, 0} y_{n, 1} x_{n, 1} \cdots y_{n, \ell} x_{n, \ell}
$$

In view of 6.14, 6.15) and (6.16), we note that

$$
z_{n} \in L_{n}(\alpha) L_{n}(\beta)^{e_{n}, 0} u_{n, 1} y_{n, 1} u_{n, 2} L_{n}(\beta)^{e_{n, 1}} u_{n, 3} \cdots y_{n, \ell} u_{n, 2 \ell} L_{n}(\beta)^{e_{n, \ell}} L_{n}(\gamma)
$$

Taking into account 6.13), we conclude that $z_{n} \in L_{\min \left\{n, e_{n, k}\right\}}(w)$. Hence $\lim z_{n}=[w]_{\mathrm{A}}$ by Corollary 6.2 . By compactness of $\bar{\Omega}_{X} \mathrm{~A}$, there is an infinite sequence $n_{1}<n_{2}<\cdots$ and there exist $x_{i}, y_{j} \in \bar{\Omega}_{X} \mathrm{~A}$ such that

$$
\lim _{h \rightarrow \infty} x_{n_{h}, i}=x_{i}, \lim _{h \rightarrow \infty} y_{n_{h}, j}=y_{j}
$$

By continuity of multiplication, we have

$$
[w]_{\mathrm{A}}=x_{0} y_{1} x_{1} \cdots y_{\ell} x_{\ell}
$$

In view of Theorem 6.4, we deduce that each $x_{i}$ belongs to $\mathrm{cl}_{\omega, \mathrm{A}}(L)$, while each $y_{j} \in \operatorname{cl}_{\omega, \mathrm{A}}\left(L^{*}\right)$. Hence, it suffices to show that each $y_{j}$ belongs to the set $\left\langle\operatorname{cl}_{\omega, \mathrm{A}}(L)\right\rangle_{\omega}$. Since $y_{j}$ is a factor of $[\beta]_{\mathrm{A}}$ by $(6.12)$ and Corollary 6.2, by [9, Lemma 4.1] we have $\operatorname{rank}\left(y_{j}\right) \leq \operatorname{rank}(\beta)<\operatorname{rank}(w)$. By the induction hypothesis, namely on the rank component, it follows that indeed $y_{j} \in$ $\left\langle\mathrm{cl}_{\omega, \mathrm{A}}(L)\right\rangle_{\omega}$, which completes the proof of Case (a).

In Case (b), we claim that there is some cycle in the graph $\Gamma$. Otherwise, the graph $\Gamma$ is linear, which means that the paths $\pi_{n}$ are all equal. We retain the notation (6.11) for their common value, but now of course all edges are red and so the edge represented vertically in (6.11) has no special meaning. As above, we choose $x_{n, i}, y_{n, j}, u_{n, j}$ satisfying (6.13) and (6.14)-(6.16) with $\left(e_{n, i}\right)_{n}$ bounded. Note that $z_{n}$ given by $(6.17)$ is actually equal to $w_{n}$. As above, there exist an infinite sequence $n_{1}<n_{2}<\cdots$ and $x_{i}, y_{j} \in \bar{\Omega}_{X} \mathrm{~A}$ such that (6.18) and (6.19) hold. Since each factor $x_{i}, y_{j}$ belongs to $\Omega_{X}^{\omega} \mathrm{A}$ by Theorem 6.4 and it has rank at most rank $(w)-1$, we conclude that so does $w$, which is absurd. This establishes the claim that $\Gamma$ contains a cycle.

Recycling once again the above notation, we may assume that there is a path 6.11 and there are indices $p, q \in\{1, \ldots, \ell\}$ such that $p<q$ and 
$\left(s_{2 p-1}, s_{2 p}\right)=\left(s_{2 q-1}, s_{2 q}\right)$. We also choose $x_{n, i}, y_{n, j}, u_{n, j}$ satisfying (6.12)(6.16). Moreover, we choose an additional word

$$
x_{n, q-1}^{\prime} \in L \cap u_{n, 2 q-2}\left(L_{n}(\beta)\right)^{*} u_{n, 2 p-1} .
$$

Note that the above intersection is nonempty, since $\varphi\left(u_{n, 2 p-1}\right)=\varphi\left(u_{n, 2 q-1}\right)$. We now choose

$$
\begin{gathered}
z_{n}=x_{n, 0} y_{n, 1} x_{n, 1} \cdots y_{n, p-1} x_{n, p-1}\left(y_{n, p} x_{n, p} \cdots y_{n, q-1} x_{n, q-1}^{\prime}\right)^{n !} \\
y_{n, p} x_{n, p} \cdots y_{n, q-1} x_{n, q-1} y_{n, q} x_{n, q} \cdots y_{n, \ell} x_{n, \ell} .
\end{gathered}
$$

We check that $z_{n} \in L_{n}(w)$, so that $\lim z_{n}=[w]_{\mathrm{A}}$ by Corollary 6.2. As above, there exist an infinite sequence $n_{1}<n_{2}<\cdots$ and $x_{i}, y_{j}, x_{q-1}^{\prime} \in$ $\bar{\Omega}_{X} \mathrm{~A}$ such that 6.18 holds and $\lim _{h \rightarrow \infty} x_{n_{h}, q-1}^{\prime}=x_{q-1}^{\prime}$. By continuity of multiplication and of the $\omega$-power, we have

$$
\begin{gathered}
{[w]_{\mathrm{A}}=x_{0} y_{1} x_{1} \cdots y_{p-1} x_{p-1}\left(y_{p} x_{p} \cdots y_{q-1} x_{q-1}^{\prime}\right)^{\omega}} \\
y_{p} x_{p} \cdots y_{q-1} x_{q-1} y_{q} x_{q} \cdots y_{\ell} x_{\ell} .
\end{gathered}
$$

By the argument in the previous case, all factors $x_{i}, y_{j}, x_{q-1}^{\prime}$ belong to $\left\langle\mathrm{cl}_{\omega, \mathrm{A}}(L)\right\rangle_{\omega}$. Hence so does $[w]_{\mathrm{A}}$.

\section{7. $\omega$-FULLNESS OF SOME APERIODIC PSEUDOVARIETIES}

A key property for a pseudovariety in our results is that of $\sigma$-fullness, where $\sigma$ is an implicit signature. It was introduced in [12, where it is shown that, if $\sigma$ is a recursively enumerable implicit signature consisting of computable operations then, for a $\sigma$-full pseudovariety $\mathrm{V}$, the closure of a regular language in the $\mathrm{V}$-free $\sigma$-algebra is computable, provided the $\sigma$ identity problem is solvable for $\mathrm{V}$. It turns out that this result applies to all the specific pseudovarieties mentioned above for the implicit signature $\omega$, consisting of multiplication and the $\omega$-power. However, the resulting algorithms are by brute force, involving the enumeration of possible proofs of membership/non-membership in the closure, and are therefore unusable in practice. Combined with the result that all pseudovarieties in question are $\omega$-reducible for the pointlike problem, this justifies the interest in establishing that the Pin-Reutenauer descriptive procedure holds for these pseudovarieties, even though the resulting description for the closure of a regular language does not by itself provide an algorithm for testing membership in the closure.

To apply Proposition 3.2 to establish that the Pin-Reutenauer procedure holds in certain pseudovarieties we need to establish that they are $\sigma$-full. We begin with some general considerations concerning this property.

Note that every locally finite pseudovariety is $\sigma$-full for every implicit signature $\sigma$. More generally, we have the following result.

Proposition 7.1. Let $\mathrm{U}$ be a locally finite pseudovariety and let $\mathrm{V}$ be a $\sigma$-full pseudovariety. Then $\mathrm{U} \vee \vee$ is $\sigma$-full. 
Proof. Denote the join $U \vee \vee$ by W. Let $\varphi: \bar{\Omega}_{X} S \rightarrow S$ be a continuous homomorphism into a finite semigroup $S$ and suppose that $w \in \bar{\Omega}_{X} S$ is such that $p_{\mathrm{W}}(w) \in \Omega_{X}^{\sigma} \mathrm{W}$. Consider the product mapping $\psi=\varphi \times p_{\mathrm{U}}$ defined by $\psi(w)=\left(\varphi(w), p_{\mathrm{U}}(w)\right)$, which is a continuous homomorphism from $\bar{\Omega}_{X} \mathrm{~S}$ into the finite semigroup $S \times \bar{\Omega}_{X} \mathrm{U}$. From $p_{\mathrm{W}}(w) \in \Omega_{X}^{\sigma} \mathrm{W}$, we deduce that $p_{\mathrm{V}}(w) \in \Omega_{X}^{\sigma} \mathrm{V}$. Since $\mathrm{V}$ is $\sigma$-full, there is some $v \in \Omega_{X}^{\sigma} \mathrm{S}$ such that $\psi(v)=\psi(w)$ and $p_{\mathrm{V}}(v)=p_{\mathrm{V}}(w)$. It follows that $\varphi(v)=\varphi(w)$ and $p_{\mathrm{U}}(v)=p_{\mathrm{U}}(w)$. As the product mapping $p_{\mathrm{U}} \times p_{\mathrm{V}}$ factors as $p_{\mathrm{W}}$ followed by an embedding $\bar{\Omega}_{X} \mathrm{~W} \hookrightarrow \bar{\Omega}_{X} \mathrm{U} \times \bar{\Omega}_{X} \mathrm{~V}$, we deduce that $p_{\mathrm{W}}(v)=p_{\mathrm{W}}(w)$.

We now prove $\omega$-fullness for several aperiodic pseudovarieties of semigroups.

Theorem 7.2. The pseudovariety A is $\omega$-full.

Proof. Let $\varphi: \bar{\Omega}_{X} S \rightarrow S$ be a continuous homomorphism onto a finite semigroup and let $w \in \bar{\Omega}_{X} \mathrm{~S}$. Suppose that $p_{\mathrm{A}}(w) \in \Omega_{X}^{\omega} \mathrm{A}$. We claim that there exists $v \in \Omega_{X}^{\omega} \mathrm{S}$ such that $\varphi(v)=\varphi(w)$ and $p_{\mathrm{A}}(v)=p_{\mathrm{A}}(w)$. We proceed by induction on $\operatorname{rank}\left(p_{\mathrm{A}}(w)\right)$. The case where $\operatorname{rank}\left(p_{\mathrm{A}}(w)\right)=0$ is obvious since then $w \in X^{+}$. We may therefore assume that $\operatorname{rank}\left(p_{\mathrm{A}}(w)\right)>0$ and that the claim holds for all elements $w^{\prime}$ of $\bar{\Omega}_{X} \mathrm{~S}$ such that $\operatorname{rank}\left(p_{\mathrm{A}}\left(w^{\prime}\right)\right)<$ $\operatorname{rank}\left(p_{\mathrm{A}}(w)\right)$.

Let $\bar{w}=x_{0} y_{1}^{\omega} x_{1} \cdots y_{r}^{\omega} x_{r}$ be a term in normal form such that $[\bar{w}]_{\mathrm{A}}=$ $p_{\mathrm{A}}(w)$. By Corollary 6.3, $w \in \operatorname{cl}\left(L_{n}(\bar{w})\right)$ for every $n$ while it is easy to see that $L_{n}(\bar{w})=L_{n}\left(x_{0}\right) L_{n}\left(y_{1}^{\omega}\right) L_{n}\left(x_{1}\right) \cdots L_{n}\left(y_{r}^{\omega}\right) L_{n}\left(x_{r}\right)$ (cf. [8, Lemma 3.2]). Therefore, the following equality holds:

$$
\operatorname{cl}\left(L_{n}(\bar{w})\right)=\operatorname{cl}\left(L_{n}\left(x_{0}\right)\right) \operatorname{cl}\left(L_{n}\left(y_{1}^{\omega}\right)\right) \operatorname{cl}\left(L_{n}\left(x_{1}\right)\right) \cdots \operatorname{cl}\left(L_{n}\left(y_{r}^{\omega}\right)\right) \operatorname{cl}\left(L_{n}\left(x_{r}\right)\right) .
$$

Hence, for every $n$ there is a factorization

$$
w=t_{n, 0} z_{n, 1} t_{n, 1} \cdots z_{n, r} t_{n, r},
$$

where each $t_{n, i} \in \operatorname{cl}\left(L_{n}\left(x_{i}\right)\right)$ and each $z_{n, j} \in \operatorname{cl}\left(L_{n}\left(y_{j}^{\omega}\right)\right)$. By taking subsequences and since, for an $\omega$-term $t$, the sets $L_{n}(t)$ form a decreasing sequence, we may as well assume that each of the sequences $\left(t_{n, i}\right)_{n}$ and $\left(z_{n, j}\right)_{n}$ converges, say respectively to $t_{i}$ and $z_{j}$ in $\bar{\Omega}_{X} \mathrm{~S}$. By Corollary $6.3, p_{\mathrm{A}}\left(t_{i}\right)=\left[x_{i}\right]_{\mathrm{A}}$ and $p_{\mathrm{A}}\left(z_{j}\right)=\left[y_{j}^{\omega}\right]_{\mathrm{A}}$. Since the $x_{i}$ are $\omega$-terms of smaller rank than that of $w$, the induction hypothesis yields the existence of $u_{i} \in \Omega_{X}^{\omega} \mathrm{S}$ such that $\varphi\left(u_{i}\right)=\varphi\left(\left[x_{i}\right]_{\mathrm{S}}\right)$ and $p_{\mathrm{A}}\left(u_{i}\right)=\left[x_{i}\right]_{\mathrm{A}}$. Hence it suffices to consider the case where $w$ is such that $p_{\mathrm{A}}(w)=\left[y^{\omega}\right]_{\mathrm{A}}$ for some $\omega$-term $y^{\omega}$ in normal form.

Since $w$ belongs to $\operatorname{cl}\left(L_{n}\left(y^{\omega}\right)\right)$ for each $n$, there exists a sequence of the form $\left(y_{n, 1} \cdots y_{n, m_{n}}\right)_{n}$ which converges to $w$ in $\bar{\Omega}_{X} S$ and such that, for each $n$, we have $y_{n, i} \in L_{n}(y)$ and $m_{n} \geq n$. For each $n \geq|S|$, we consider the Cayley graph of the subsemigroup of $S$ generated by $X_{n}=\left\{\varphi\left(y_{n, i}\right)\right.$ : $\left.i=1, \ldots, m_{n}\right\}$ with respect to this generating set, and the path $\gamma_{n}$ from the vertex $\varphi\left(y_{n, 1} \cdots y_{n,|S|}\right)$ to $\varphi\left(y_{n, 1} \cdots y_{n, m_{n}}\right)$ determined by the successive multiplication by $\varphi\left(y_{n,|S|+1}\right)$ through $\varphi\left(y_{n, m_{n}}\right)$. Since $S$ is finite we may assume that the set of generators $X_{n}$ (and therefore the associated Cayley 
graph), as well as the set of edges used in the path $\gamma_{n}$ and its start and end vertices are constant. We may then choose a path $\gamma$ using some of those edges from $\varphi\left(y_{n, 1} \cdots y_{n,|S|}\right)$ to $\varphi\left(y_{n, 1} \cdots y_{n, m_{n}}\right)$ of length $\ell \leq|S|$. For each $n$, we choose $i_{n, 1}, \ldots, i_{n, \ell} \in\left\{|S|+1, \ldots, m_{n}\right\}$ such that the path $\gamma$ corresponds to successive multiplication by $\varphi\left(y_{n, i_{n, 1}}\right), \ldots, \varphi\left(y_{n, i_{n, \ell}}\right)$.

By compactness, we may assume that each of the sequences $\left(y_{n, j}\right)_{n}$ (with $j=1, \ldots,|S|)$ and $\left(y_{n, i_{n, k}}\right)_{n}$ (with $k=1, \ldots, \ell$ ) converges, say respectively to $z_{1}, \ldots, z_{m} \in \bar{\Omega}_{X} \mathrm{~S}$, where $m=|S|+\ell$. Note that, by construction, $\varphi\left(z_{1} \cdots z_{m}\right)=\varphi(w)$. Since $m \geq|S|$, there exist indices $i$ and $j$ such that $\varphi(w)=\varphi\left(z_{1} \cdots z_{i-1}\left(z_{i} \cdots z_{j-1}\right)^{\omega} z_{j} \cdots z_{m}\right)$. On the other hand, since, for every $n$, we have $y_{n, k} \in L_{n}(y)$, Corollary 6.3 implies that $p_{\mathrm{A}}\left(z_{k}\right)=[y]_{\mathrm{A}}$ for every $k \in\{1, \ldots, m\}$. Since $\operatorname{rank}(y)<\operatorname{rank}(w)$, by the induction hypothesis there exists a $v_{k} \in \Omega_{X}^{\omega} \mathrm{S}$ such that $\varphi\left(v_{k}\right)=\varphi\left(z_{k}\right)$ and $p_{\mathrm{A}}\left(v_{k}\right)=[y]_{\mathrm{A}}(k=$ $1, \ldots, m)$. Finally, it suffices to take $v=v_{1} \cdots v_{i-1}\left(v_{i} \cdots v_{j-1}\right)^{\omega} v_{j} \cdots v_{m}$.

The remainder of the section is dedicated to proving that several familiar aperiodic pseudovarieties are $\omega$-full.

By the content $c(w)$ of a pseudoword $w$ we mean the set of all its factors of the form $a$, where $a$ is a letter.

Theorem 7.3. Every subpseudovariety of J is $\omega$-full.

Proof. Let $\mathrm{V}$ be a subpseudovariety of $\mathrm{J}$. We have to show that, given a pseudoword $w \in \bar{\Omega}_{X} \mathrm{~S}$ such that $p_{\mathrm{V}}(w) \in \Omega_{X}^{\omega} \mathrm{V}$, and a continuous homomorphism $\varphi: \bar{\Omega}_{X} S \rightarrow S$ onto a finite semigroup $S$, there exists $v \in \Omega_{X}^{\omega} S$ such that

$$
\varphi(v)=\varphi(w) \text { and } p_{\vee}(v)=p_{\vee}(w)
$$

By [1, Thm. 8.1.11], one can factorize $w$ as a finite product of elements of $\bar{\Omega}_{X} \mathrm{~S}$, each of them having the following property: either it is a word, or for all $u \in X^{+}$, it contains as a subword either all or none of the words of $u^{+}$. Therefore, one may assume that $w$ itself has this property.

If $w$ is a word, then $w \in \Omega_{X}^{\omega} \mathrm{S}$ and there is nothing to prove. Otherwise, let $u$ be a word with the same content as $w$, so that $u^{k}$ is a subword of $w$ for all $k>0$. Consider, for each $k>0$, a factorization $w=w_{1} \cdots w_{k} w_{k}^{\prime}$, where $u$ is a subword of $w_{i}$. Since $S$ is finite, there exist integers $n, p>0$ such that $\varphi\left(w_{1} \cdots w_{n}\right)=\varphi\left(w_{1} \cdots w_{n} w_{n+1} \cdots w_{n+p}\right)$. Let us also choose, for $1 \leq i \leq n+p$, a word $v_{i}$ of content $c(u)$ such that $\varphi\left(v_{i}\right)=\varphi\left(w_{i}\right)$, and similarly a word $v_{n}^{\prime}$ of content $c(u)$ such that $\varphi\left(v_{n}^{\prime}\right)=\varphi\left(w_{n}^{\prime}\right)$; this is possible since it is well known that the content function is continuous and $X^{+}$is dense in $\bar{\Omega}_{X} \mathrm{~S}$. We put $v=v_{1} \cdots v_{n}\left(v_{n+1} \cdots v_{n+p}\right)^{\omega} v_{n}^{\prime}$. Then $\mathrm{J}$ satisfies 
$v=u^{\omega}=w$, whence so does $\mathrm{V}$, and the following equalities hold

$$
\begin{array}{rlrl}
\varphi(v) & =\varphi\left(v_{1} \cdots v_{n}\left(v_{n+1} \cdots v_{n+p}\right)^{\omega} v_{n}^{\prime}\right) & \\
& =\varphi\left(w_{1} \cdots w_{n}\left(w_{n+1} \cdots w_{n+p}\right)^{\omega} w_{n}^{\prime}\right) & & \text { by the choice of } v_{i} \text { and } v_{n}^{\prime} . \\
& =\varphi\left(w_{1} \cdots w_{n} w_{n}^{\prime}\right) & & \text { by the choice of } n, p . \\
& =\varphi(w) . &
\end{array}
$$

Therefore, this choice of $v$ fulfills condition (7.1).

In turn, the following is an application of well-developed techniques concerning the pseudovariety $\mathrm{R}$ of all finite R-trivial semigroups.

Theorem 7.4. The pseudovariety $\mathrm{R}$ is $\omega$-full.

Proof. Let $w$ be an element of the free profinite monoid $\left(\bar{\Omega}_{X} \mathrm{~S}\right)^{1}$ and $\varphi$ : $\left(\bar{\Omega}_{X} S\right)^{1} \rightarrow S$ be a continuous homomorphism into a finite monoid $S$, and assume that $p_{\mathrm{R}}(w) \in \Omega_{X}^{\omega} \mathrm{R}$. Since $\mathrm{R}$ is generated by monoids, it suffices to show that there exists $v \in\left(\Omega_{X}^{\omega} \mathrm{S}\right)^{1}$ such that

$$
\varphi(v)=\varphi(w) \text { and } p_{\mathrm{R}}(v)=p_{\mathrm{R}}(w) .
$$

To prove this, we proceed by induction on $|c(w)|$. For the case $|c(w)|=0$, that is $w=1$, there is nothing to be done, so we assume that $|c(w)|>0$.

Consider the following factorization of $w$, which is obtained by iterating the left basic factorization on the right [14]:

$$
w=w_{1} a_{1} w_{2} a_{2} \cdots w_{k} a_{k} w_{k}^{\prime}
$$

where, for each $i, c(w)=c\left(w_{i}\right) \uplus\left\{a_{i}\right\}$, and $k$ is either the largest possible, that is $c\left(w_{k}^{\prime}\right) \varsubsetneqq c(w)$, or there is no upper bound on the values of $k$ for which there is such a factorization, which is equivalent to $p_{\mathrm{R}}(w)$ being idempotent. The factorization is unique in the following sense: the sequences $a_{1}, a_{2}, \ldots$ and $p_{\mathrm{R}}\left(w_{1}\right), p_{\mathrm{R}}\left(w_{2}\right), \ldots$ are completely determined by $w$, and so is each $p_{\mathrm{R}}\left(w_{k}^{\prime}\right)$. In particular, $p_{\mathrm{R}}\left(w_{k}^{\prime}\right)$ completely determines $a_{k+1}, a_{k+2}, \ldots$ and $p_{\mathrm{R}}\left(w_{k+1}\right), p_{\mathrm{R}}\left(w_{k+2}\right), \ldots$. Since $p_{\mathrm{R}}(w)$ is an $\omega$-word, by [15, Theorem 4.4] there is only a finite number of possible values for the remainders $p_{\mathrm{R}}\left(w_{k}^{\prime}\right)$. Hence, in case $p_{\mathrm{R}}(w)$ is idempotent, there are integers $p$ and $q$ such that $p<q$ and $\mathrm{R}$ satisfies $w_{q}^{\prime}=w_{p}^{\prime}=w_{p+1} a_{p+1} \cdots w_{q} a_{q} w_{q}^{\prime}$. We then replace our choice of $k$ by $k=p+(|S|+1)(q-p)$.

By the induction hypothesis, since each $w_{i}$ has smaller content than $w$ and $p_{\mathrm{R}}\left(w_{i}\right) \in \Omega_{X}^{\omega} \mathrm{R}$ by [15, Lemma 2.2], there exists $v_{i} \in \Omega_{X}^{\omega} \mathrm{S}$ such that $\varphi\left(v_{i}\right)=\varphi\left(w_{i}\right)$ and the pseudoidentity $v_{i}=w_{i}$ holds in R.

In case $p_{\mathrm{R}}(w)$ is not idempotent, the induction hypothesis also yields the existence of $v_{k}^{\prime} \in \Omega_{X}^{\omega} \mathrm{S}$ such that $\varphi\left(v_{k}^{\prime}\right)=\varphi\left(w_{k}^{\prime}\right)$ and R satisfies $v_{k}^{\prime}=w_{k}^{\prime}$. Then we simply take $v=v_{1} a_{1} \cdots v_{k} a_{k} v_{k}^{\prime}$ and observe that 7.2 holds for this choice of $v$. 
Consider next the case where $p_{\mathrm{R}}(w)$ is idempotent. Let

$$
\begin{aligned}
x_{0}= & v_{1} a_{1} \cdots v_{p} a_{p} \\
x_{i}= & v_{p+(q-p)(i-1)+1} a_{p+(q-p)(i-1)+1} \cdots v_{q+(q-p)(i-1)} a_{q+(q-p)(i-1)} \\
& (i=1, \ldots,|S|+1) .
\end{aligned}
$$

There exist indices $r$ and $s$ such that $1 \leq r<s \leq|S|+1$ and $\varphi\left(x_{1} \cdots x_{r}\right)=$ $\varphi\left(x_{1} \cdots x_{s}\right)$. As in the proof of Theorem 7.3 , we may choose a word $y$ with the same content as $w$ such that $\varphi(y)=\varphi\left(w_{q+(q-p)(s-1)}^{\prime}\right)$. We let $v=$ $x_{0} x_{1} \cdots x_{r}\left(x_{r+1} \cdots x_{s}\right)^{\omega} y$. It is then easy to verify that $v$ satisfies (7.2).

By Propositions 5.2 and $4.4(i)$, since $\mathrm{A}$ is $\omega$-factorial and both $\mathrm{A}$ and $\mathrm{R}$ are $\omega$-full, we deduce that property (5.1) holds for $\mathrm{V}=\mathrm{R}$ and $\sigma=\omega$. Note that $\mathrm{R}$ is not $\omega$-factorial. For instance, over the two-letter alphabet $X=\{a, b\}$, we have $(a b)^{\omega} u=(a b)^{\omega}$ for every $u \in \bar{\Omega}_{X} \mathrm{R}$, while $\bar{\Omega}_{X} \mathrm{R}$ is uncountable. It follows that condition $(c)$ cannot be omitted from the hypothesis of Proposition 5.2 .

Theorem 7.5. The pseudovariety DA is $\omega$-full.

Proof. The proof follows the same lines as that of Theorem 7.4 using central basic factorizations [2] instead of left basic factorizations. Let $w$ be an element of $\left(\bar{\Omega}_{X} \mathrm{~S}\right)^{1}$ and $\varphi:\left(\bar{\Omega}_{X} \mathrm{~S}\right)^{1} \rightarrow S$ be a continuous homomorphism onto a finite monoid $S$, and suppose that $p_{\mathrm{DA}}(w) \in\left(\Omega_{X}^{\omega} \mathrm{DA}\right)^{1}$. We claim that there exists $v \in\left(\Omega_{X}^{\omega} \mathrm{S}\right)^{1}$ such that

$$
\varphi(v)=\varphi(w) \text { and } p_{\mathrm{DA}}(v)=p_{\mathrm{DA}}(w) .
$$

We proceed by induction on $|c(w)|$. The case $|c(w)|=0$ being trivial, we assume that $|c(w)|>0$.

By iterating the central basic factorization of $w$, we obtain a sequence of factorizations of the form

$$
w=w_{1} a_{1} w_{2} a_{2} \cdots w_{k} a_{k} w_{k}^{\prime} b_{k} w_{k}^{\prime \prime} \cdots b_{2} w_{2}^{\prime \prime} b_{1} w_{1}^{\prime \prime}
$$

where, for each $i, c\left(w_{i}\right) \uplus\left\{a_{i}\right\}=c(w)=c\left(w_{i}^{\prime \prime}\right) \uplus\left\{b_{i}\right\}$, and one of the following conditions holds:

(i) $k$ is the largest possible, that is $c\left(w_{k}^{\prime}\right) \varsubsetneqq c(w)$ or there is a factorization of one of the forms $w_{k}^{\prime}=z_{1} d_{1} z_{2}$ or $w_{k}^{\prime}=z_{1} d_{1} z_{2} d_{2} z_{3}$ with $d_{i} \in X$ and $c\left(z_{i}\right) \varsubsetneqq c(w)$ for all $i$;

(ii) there is no upper bound on the values of $k$ for which there is such a factorization, which is equivalent to $p_{\mathrm{DA}}(w)$ being idempotent.

The factorization is unique in the sense that each of the (possibly finite) sequences $\left(p_{\mathrm{DA}}\left(w_{i}\right)\right)_{i},\left(a_{i}\right)_{i},\left(p_{\mathrm{DA}}\left(w_{i}^{\prime}\right)\right)_{i},\left(b_{i}\right)_{i}$, and $\left(p_{\mathrm{DA}}\left(w_{i}^{\prime \prime}\right)\right)_{i}$ is completely determined by $w$. Since $p_{\mathrm{DA}}(w)$ is an $\omega$-word, by [27, Theorem 4.3] there is only a finite number of possible values for the central remainders $p_{\mathrm{DA}}\left(w_{k}^{\prime}\right)$. Hence, in case $p_{\text {DA }}(w)$ is idempotent, there are integers $p$ and $q$ such that $p<q$ and DA satisfies $w_{q}^{\prime}=w_{p}^{\prime}=w_{p+1} a_{p+1} \cdots w_{q} a_{q} w_{q}^{\prime} b_{q} w_{q}^{\prime \prime} \cdots b_{p+1} w_{p+1}^{\prime \prime}$. We then choose $k=q$. 
Since each of the factors $w_{i}$ and $w_{i}^{\prime \prime}$ has smaller content than $w$, the induction hypothesis yields the existence of $v_{i}, v_{i}^{\prime \prime} \in \Omega_{X}^{\omega} \mathrm{S}$ such that $\varphi\left(v_{i}\right)=$ $\varphi\left(w_{i}\right), \varphi\left(v_{i}^{\prime \prime}\right)=\varphi\left(w_{i}^{\prime \prime}\right), p_{\mathrm{DA}}\left(v_{i}\right)=p_{\mathrm{DA}}\left(w_{i}\right)$ and $p_{\mathrm{DA}}\left(v_{i}^{\prime \prime}\right)=p_{\mathrm{DA}}\left(w_{i}^{\prime \prime}\right)(i=$ $1, \ldots, k)$.

In Case $(i), w_{k}^{\prime}$ has a finite factorization $w_{k}^{\prime}=y_{1} \cdots y_{r}$ such that $c\left(y_{i}\right) \varsubsetneqq$ $c(w)(i=1, \ldots, r)$, where $r$ is 3 or 5 . We may apply the induction hypothesis to each $y_{i}$ to obtain $t_{i} \in \Omega_{X}^{\omega} \mathrm{S}$ such that $\varphi\left(t_{i}\right)=\varphi\left(y_{i}\right)$ and $p_{\mathrm{DA}}\left(t_{i}\right)=p_{\mathrm{DA}}\left(y_{i}\right)$ $(i=1, \ldots, r)$; in this case, we let $v_{k}^{\prime}=t_{1} \cdots t_{r}$. We may now take

$$
v=v_{1} a_{1} \cdots v_{k} a_{k} v_{k}^{\prime} b_{k} v_{k}^{\prime \prime} \cdots b_{1} v_{1}^{\prime \prime}
$$

and check that (7.3) holds for this choice of $v$.

It remains to treat the case where $p_{\mathrm{DA}}(w)$ is idempotent. Let $m=q-p$ and

$$
\begin{aligned}
x_{0} & =v_{1} a_{1} \cdots v_{p} a_{p} \\
x_{0}^{\prime \prime} & =b_{p} v_{p}^{\prime \prime} \cdots b_{1} v_{1}^{\prime \prime} \\
x_{i+1} & =v_{p+m i+1} a_{p+m i+1} \cdots v_{q+m i} a_{q+m i} \\
x_{i+1}^{\prime \prime} & =b_{q+m i} v_{q+m i}^{\prime \prime} \cdots b_{p+m i+1} v_{p+m i+1}^{\prime \prime},
\end{aligned}
$$

for $i=0, \ldots,|S|$. By the pigeonhole principle, there exist indices $r, s, r^{\prime \prime}$, and $s^{\prime \prime}$ such that $1 \leq r<s \leq|S|+1,1 \leq r^{\prime \prime}<s^{\prime \prime} \leq|S|+1, \varphi\left(x_{1} \cdots x_{r}\right)=$ $\varphi\left(x_{1} \cdots x_{s}\right)$, and $\varphi\left(x_{r^{\prime \prime}}^{\prime \prime} \cdots x_{1}^{\prime \prime}\right)=\varphi\left(x_{s^{\prime \prime}}^{\prime \prime} \cdots x_{1}^{\prime \prime}\right)$. Let $n$ be an index such that $n>\max \left\{s, s^{\prime \prime}\right\}$ and choose any word $y$ with the same content as $w$ such that

$\varphi(y)=\varphi\left(w_{q+m n}^{\prime}\right)$; this is possible since it is well known that the content function is continuous and $X^{+}$is dense in $\bar{\Omega}_{X} \mathrm{~S}$. We let

$$
\begin{aligned}
& v=x_{0} x_{1} \cdots x_{r}\left(x_{r+1} \cdots x_{s}\right)^{\omega} x_{s+1} \cdots x_{n} \\
& \cdot y x_{n}^{\prime \prime} \cdots x_{s^{\prime \prime}+1}^{\prime \prime}\left(x_{s^{\prime \prime}}^{\prime \prime} \cdots x_{r^{\prime \prime}+1}^{\prime \prime}\right)^{\omega} x_{r^{\prime \prime}}^{\prime \prime} \cdots x_{1}^{\prime \prime} x_{0}^{\prime \prime} .
\end{aligned}
$$

One can then verify that $v$ satisfies $(7.3)$.

\section{ACKNOWLEDGMENTS}

Work partly supported by the AutoMathA programme of the European Science Foundation and by the PESSOA French-Portuguese project EgideGrices 11113YM, Automata, profinite semigroups and symbolic dynamics.

The work of the first two authors was supported, in part, by the European Regional Development Fund, through the programme COMPETE, and by the Portuguese Government through FCT - Fundação para a Ciência e a Tecnologia, respectively under the projects PEst-C/MAT/UI0144/2011 and PEst-C/MAT/UI0013/2011.

The work of the first two authors was also partly supported by the FCT and the project PTDC/MAT/65481/2006 which was partly funded by the European Community Fund FEDER, and by the INTAS grant \#99-1224.

The work of the third author was partly supported by ANR 2010 BLAN 020201 FREC. 


\section{REFERENCES}

1. J. Almeida, Finite semigroups and universal algebra, World Scientific, Singapore, 1995.

2. _ A syntactical proof of locality of DA, Int. J. Algebra Comput. 6 (1996), 165177.

3. __ Some algorithmic problems for pseudovarieties, Publ. Math. Debrecen 54 Suppl. (1999), 531-552.

4. - Finite semigroups: an introduction to a unified theory of pseudovarieties, Semigroups, Algorithms, Automata and Languages (Singapore) (G. M. S. Gomes, J.-É. Pin, and P. V. Silva, eds.), World Scientific, 2002, pp. 3-64.

5. __ Profinite semigroups and applications, Structural Theory of Automata, Semigroups, and Universal Algebra (New York) (Valery B. Kudryavtsev and Ivo G. Rosenberg, eds.), NATO Science Series II: Mathematics, Physics Chemistry, vol. 207, Springer, 2005, Proceedings of the NATO Advanced Study Institute on Structural Theory of Automata, Semigroups and Universal Algebra, Montréal, Québec, Canada, 7-18 July 2003.

6. J. Almeida, J. C. Costa, and M. Zeitoun, On the reducibility property of some pseudovarieties, In preparation.

7. _ Complete reducibility of systems of equations with respect to $\mathrm{R}$, Portugal. Math. 64 (2007), 445-508.

8. __ McCammond normal forms for free aperiodic semigroups revisited, Tech. Report CMUP 2012-3, Univ. Porto, 2012, Submitted.

9. __ Iterated periodicity over finite aperiodic semigroups, European J. Combin. 37 (2014), 115-149.

10. J. Almeida and M. Delgado, Sur certains systèmes d'équations avec contraintes dans un groupe libre, Portugal. Math. 56 (1999), no. 4, 409-417.

11. __ Sur certains systèmes d'équations avec contraintes dans un groupe libreaddenda, Portugal. Math. 58 (2001), no. 4, 379-387.

12. J. Almeida and B. Steinberg, On the decidability of iterated semidirect products and applications to complexity, Proc. London Math. Soc. 80 (2000), 50-74.

13. _ Syntactic and global semigroup theory, a synthesis approach, Algorithmic Problems in Groups and Semigroups (J. C. Birget, S. W. Margolis, J. Meakin, and M. V. Sapir, eds.), Birkhäuser, 2000, pp. 1-23.

14. J. Almeida and P. Weil, Free profinite $\mathcal{R}$-trivial monoids, Int. J. Algebra Comput. 7 (1997), 625-671.

15. J. Almeida and M. Zeitoun, An automata-theoretic approach to the word problem for $\omega$-terms over $R$, Theor. Comp. Sci. 370 (2007), 131-169.

16. A. W. Anissimow and F. D. Seifert, Zur algebraischen Charakteristik der durch kontext-freie Sprachen definierten Gruppen, Elektron. Informationsverarbeit. Kybernetik 11 (1975), no. 10-12, 695-702.

17. C. J. Ash, Inevitable graphs: a proof of the type II conjecture and some related decision procedures, Int. J. Algebra Comput. 1 (1991), 127-146.

18. K. Auinger, A new proof of the Rhodes type II conjecture, Int. J. Algebra Comput. 14 (2004), no. 5-6, 551-568.

19. K. Auinger and B. Steinberg, A constructive version of the Ribes-Zalesskiu product theorem, Math. Z. 250 (2005), 287-297.

20. J. Berstel, Transductions and context-free languages, Teubner Verlag, 1979, The first four chapters are available through http://www-igm.univ-mlv.fr/〜berstel/ LivreTransductions/LivreTransductions.html.

21. J. C. Costa and C. Nogueira, Complete reducibility of the pseudovariety LSl, Int. J. Algebra Comput. 19 (2009), no. 2, 247-282.

22. K. Henckell, Pointlike sets: the finest aperiodic cover of a finite semigroup, J. Pure Appl. Algebra 55 (1988), no. 1-2, 85-126. 
23. K. Henckell, S. W. Margolis, J.-É. Pin, and J. Rhodes, Ash's type II theorem, profinite topology and Mal'cev products. I, Int. J. Algebra Comput. 1 (1991), no. 4, 411-436.

24. K. Henckell, J. Rhodes, and B. Steinberg, Aperiodic pointlikes and beyond, Int. J. Algebra Comput. 20 (2010), no. 2, 287-305.

25. B. Herwig and D. Lascar, Extending partial automorphisms and the profinite topology on free groups, Trans. Amer. Math. Soc. 352 (2000), no. 5, 1985-2021.

26. J. P. McCammond, Normal forms for free aperiodic semigroups, Int. J. Algebra Comput. 11 (2001), no. 5, 581-625.

27. A. Moura, The word problem for $\omega$-terms over DA, Theor. Comp. Sci. (2011), 65566569.

28. C. V. Nogueira, Propriedades algorítmicas envolvendo a pseudovariedade LSl, Ph.D. thesis, Univ. Minho, 2010.

29. K. Numakura, Theorems on compact totally disconnected semigroups and lattices, Proc. Amer. Math. Soc. 8 (1957), 623-626.

30. J.-É. Pin, Varieties of formal languages, Foundations of Computer Science, Plenum Publishing, 1986.

31. __ Topologies for the free monoid, J. Algebra 137 (1991), no. 2, 297-337.

32. J.-É. Pin and Ch. Reutenauer, A conjecture on the Hall topology for the free group, Bull. London Math. Soc. 23 (1991), 356-362.

33. J. Rhodes and B. Steinberg, The q-theory of finite semigroups, Springer Monographs in Mathematics, Springer, 2009.

34. L. Ribes and P. A. Zalesskiı̌, On the profinite topology on a free group, Bull. London Math. Soc. 25 (1993), 37-43.

35. B. Steinberg, On pointlike sets and joins of pseudovarieties, Int. J. Algebra Comput. 8 (1998), 203-231.

36. _ On algorithmic problems for joins of pseudovarieties, Semigroup Forum 62 (2001), 1-40.

(J. Almeida) Centro de Matemática e Departamento de Matemática, Faculdade de Ciências, Universidade do Porto, Rua do Campo Alegre, 687, 4169-007 Porto, Portugal. JALMEIDA@FC.UP.PT

(J. C. Costa) Centro de Matemática e Departamento de Matemática e ApliCaÇões, Universidade do Minho, Campus de Gualtar, 4700-320 Braga, PortuGAL. JCOSTA@MATH.UMINHO.PT

(M. Zeitoun) LaBRI, Université de Bordeaux \& CNRS UMR 5800. 351 Cours de la Libération, 33405 Talence Cedex, France. mz@labri.fr 